\title{
artigo
}

Medeiros, A.C.L.V.; Ramos, W.T.; Andrade, M.M.; Santos, H.; Araújo, M.S.; Santos, G.S.

A sexualidade na adolescência e a importância da educação em saúde na escola: relato de experiência

\section{A sexualidade na adolescência e a importância da educação em saúde na escola: relato de experiência}

\author{
Sexuality in adolescence and the importance of health education in school: experience report
}

La sexualidad en la adolescencia y la importancia de la educación para la salud en la escuela: informe de experiencia

\begin{abstract}
RESUMO
Objetiva-se relatar uma experiência sobre a importância da educação em saúde para conscientização da sexualidade na adolescência. Trata-se de um estudo qualitativo, descritivo, formato relato de experiência, de acadêmicos de fisioterapia da Universidade Federal de Sergipe em uma escola de Lagarto (SE), baseando-se no método do Arco de Charles Magueréz e no Programa Saúde na Escola, com apoio da escola e equipe de saúde de referência. Foram utilizadas questões norteadoras com a construção de cartaz coletivo, apresentações, vídeos, peças anatômicas e peça teatral para abordar a saúde sexual e reprodutiva, a sexualidade e abuso e/ou violência sexual. Com o estudo, observou-se que os adolescentes refletiram e avaliaram suas concepções sobre a sexualidade, encarando-a com mais cuidado e respeito, favorecendo uma mudança em sua realidade. Aos acadêmicos da fisioterapia possibilitou entender a importância dessa temática na educação em saúde, favorecendo uma ampliação do olhar em sua prática profissional. Assim, pode-se concluir que tal abordagem contribui para mudanças efetivas na vida dos adolescentes e demonstra a necessidade do desenvolvimento de mais ações e estudos acerca da sexualidade pelos profissionais de saúde, preparando-os para atuar próximos às necessidades da sociedade.
\end{abstract}

DESCRITORES: Adolescente; Sexualidade; Abuso Sexual na Infância; Educação em Saúde.

\section{ABSTRACT}

Aim to report an experience about the importance of health education on awareness about adolescence's sexuality. Method: a qualitative, descriptive and experience report study developed by physiotherapy students of the Sergipe Federal University in a school of Lagarto (SE), based on the method of the Charles Magueréz Arch and the School Health Program, with support from the school and reference health team. We used guiding questions to construct a collective poster, presentations, videos, anatomical pieces and a play to talk about sexual and reproductive health, sexuality and sexual abuse and/or sexual violence. With the study, it was observed that the adolescents reflected and evaluated their conceptions about sexuality, facing it with more care and respect, favoring a change in their reality. Physiotherapy students made it possible to understand the importance of this theme in health education, favoring a broader view of their professional practice. Thus, it can be concluded that such an approach contributes to effective changes in the lives of adolescents and demonstrates the need for the development of more actions and studies on sexuality by health professionals, preparing them to act close to the needs of society.

DESCRIPTORS: Adolescent; Sexuality; Child Abuse, Sexual; Health Education.

\section{RESUMEN}

El objetivo es relatar una experiencia sobre la importancia de la educación en salud para concienciar sobre la sexualidad en la adolescencia. Se trata de un estudio cualitativo, descriptivo, formato de relato de experiencia, de estudiantes de fisioterapia de la Universidad Federal de Sergipe en un colegio de Lagarto (SE), basado en el método del Arco de Carlos Magueréz y el Programa Salud en la Escuela, con el apoyo de la escuela y un equipo de salud de referencia. Se utilizaron preguntas orientadoras con la construcción de un cartel colectivo, presentaciones, videos, obras de teatro anatómicas y una obra de teatro para abordar la salud sexual y reproductiva, la sexualidad y el abuso y / o violencia sexual. Con el estudio se observó que las adolescentes reflexionaron y evaluaron sus concepciones sobre la sexualidad, enfrentándola con más cuidado y respeto, favoreciendo un cambio en su realidad. Los estudiantes de fisioterapia permitieron comprender la importancia de este tema en la educación para la salud, favoreciendo una visión más amplia de su práctica profesional. Así, se puede concluir que tal abordaje contribuye a cambios efectivos en la vida de los adolescentes y demuestra la necesidad de desarrollar más acciones y estudios sobre sexualidad por parte de los profesionales de la salud, preparándolos para actuar cerca de las necesidades de la sociedad.

DESCRIPTORES: Adolescente; Sexualidad; Abuso Sexual Infantil; Educación en Salud.

RECEBIDO EM: 30/01/2021 APROVADO EM: 01/03/2021 


\section{Ana Catarina Leite Véras Medeiros}

Professora substituta - Universidade Federal de Sergipe (UFS), fisioterapeuta formada pela Universidade Federal da Paraíba, Sanitarista e Mestre em ciências da saúde pelo Centro de Pesquisas Aggeu Magalhães.

ORCID: 0000-0001-7093-9556

\section{Willamis Tenório Ramos}

Graduando de Fisioterapia - Universidade Federal de Sergipe (UFS).

ORCID: 0000-0002-6037-2856

\section{Mariana Moreira Andrade}

Graduanda de Fisioterapia - Universidade Federal de Sergipe (UFS).

ORCID: 0000-0002-6357-8024

\section{Hércules dos Santos}

Graduando de Fisioterapia - Universidade Federal de Sergipe (UFS). ORCID: 0000-0002-4188-5736

\section{Marcelo Santos de Araújo}

Graduando de Fisioterapia - Universidade Federal de Sergipe (UFS).

ORCID: 0000-0003-0469-7012

\section{Glaucimária Santana Santos}

Graduanda de Fisioterapia - Universidade Federal de Sergipe (UFS). ORCID: 0000-0002-1362-3210

\section{INTRODUÇÃO}

A constituição de 1988 estabelece vários direitos à população brasileira, entre eles, o direito à Saúde e a Educação. Outra conquista histórica, fruto da luta pela cidadania e pelos Direitos Humanos, reconhecidos em leis nacionais e documentos internacionais, são os direitos à saúde sexual e à saúde reprodutiva. Com o surgimento e fortalecimento do Sistema Único de Saúde (SUS) várias são as estratégias criadas para garantir a saúde da população. ${ }^{1,2}$.

Atrelado a isso, uma discussão cada vez mais importante, no âmbito mundial, é a necessidade de integrar a saúde à escola. Essa interação possibilita um caminho para evitar agravos e promover a saúde e qualidade de vida, pois a escola fornece importantes elementos para capacitar o cidadão para uma vida saudável. Ela é um espaço de relações e desenvolvimento crítico e político que ultrapassam suas fronteiras e assim, um local privilegiado para práticas de promoção, prevenção e de educação em saúde. Apesar disso, construir essas práticas pedagógicas é um grande desafio frente às demandas que as escolas enfren- tam e por isso, deve-se ter um programa de saúde na escola, com apoio das equipes de Saúde da Família, inserido e integrado no cotidiano e na cultura escolar, indo além dos limites da escola. ${ }^{1}$

Com esse intuito, foi criado em 2007, o Programa Saúde na Escola (PSE) que tem como público crianças, adolescentes e jovens no ambiente escolar e atua de forma intersetorial, seguindo os princípios e diretrizes do SUS, promovendo a articulação de saberes e a participação de alunos, pais, comunidade escolar e sociedade em geral ao tratar a saúde e educação de forma integral. As ações em saúde previstas no PSE envolvem atenção, promoção, prevenção e assistência, e devem planejar junto às escolas, a inclusão das temáticas de educação em saúde no seu projeto político pedagógico de forma que estejam voltados ao direito à vida. Entre uma das temáticas que devem ser abordadas estão as ações de promoção da saúde sexual e reprodutiva. ${ }^{3}$

A sexualidade é constitutiva dos sujeitos desde a infância em se tratando da adolescência, demanda cuidado e atenção, pois é uma fase complexa e dinâmica dos 10 a 19 anos e que envolve mudanças físicas e emocionais que começam a se acen- tuar, sobretudo pelo processo hormonal, pelo corpo, comportamento e interação social e que podem afetar a personalidade e a forma de agir na sociedade. $\mathrm{Na}$ adolescência, a sexualidade se relaciona a um campo de descobertas e experiências que implicam a tomada de decisões, requerendo responsabilidade e o exercício da autonomia. ${ }^{4}$

É nessa fase que podem ocorrer os casos de abuso e/ou violência sexual que é uma violação dos direitos sexuais de um indivíduo, sem seu conscentimento e por isso, necessita de muita atenção por parte das organizações que promovem o bem estar da criança e do adolescente, estima-se que, a cada oito jovens no mundo, um tem relato de violência sexual. No Brasil, de acordo com a Pesquisa Nacional de Saúde do Escolar (PeNSE) de 2015, é o segundo tipo de violência mais frequente e $4 \%$ da população infanto-juvenil já sofreu violência sexual, com ou sem contato físico, tendo maior prevalência no sexo feminino (10-20\%). ${ }^{5}$

Sabendo-se que o processo de educação sexual ocorre, informalmente, a partir das relações com o ambiente, tendo a família como referência, e, formalmente, como 
prática pedagógica, nas escolas e instituições sociais. Apesar dos avanços na área, a sociedade ainda tem preconceito e a sexualidade permanece sendo considerada um tabu. Os pais, muitas vezes, têm dificuldade de abordar esse assunto seja por resistência devido à cultura ou à religião ou falta de informação sobre o assunto e a escola passa a ser um local privilegiado para garantia desses direitos sexuais e reprodutivos. ${ }^{6,7}$

Defendida na Lei de Diretrizes e Bases da Educação Nacional (LDB), a sexualidade deve perpassar todas as disciplinas, com visão ampla, respeitando o caráter cultural, social e histórico, para que os alunos desenvolvam e exerçam sua sexualidade com prazer, saúde e responsabilidade. Apesar disso, ainda se questiona a realização e efetividade dessas práticas, pois pesquisas sobre comportamentos sexuais de adolescentes evidenciam que esse público tem colocado sua saúde em risco. Considera assim, a escola como favorável para a promoção da saúde e os debates sobre sexualidade devem ser realizados conjuntamente entre educadores, pais e profissionais da saúde, pois a falta de informações sobre o tema contribui para a vulnerabilidade dos jovens, aumentando a chance de comportamentos sexuais de risco. ${ }^{7,9}$,

Assim, a educação em saúde constitui uma estratégia de atuação diferenciada e dinâmica para produção de autonomia dos sujeitos, e quando integrada a equipe de saúde e a escola, aproxima o adolescente da temática, capacita os professores nessa lacuna do conhecimento e prepara os profissionais de saúde para atuar próximo à realidade social. Para isso, deve ser realizada com conteúdos programados para o meio social inserido, com respeito às peculiaridades culturais, possibilitando autoconhecimento, aquisição de informações fundamentadas e melhorias atuais e a longo prazo na saúde desses adolescentes. ${ }^{8,9,10}$

Dessa forma, o PSE torna-se um importante meio para atingir esses objetivos, ao integrar escola e equipe de saúde e trazer a família para o debate e cuidado relacionado às principais problemáticas que afetam diretamente o modo de vi-
Assim, a educação em saúde constitui uma estratégia de atuação diferenciada

e dinâmica para

produção de autonomia dos sujeitos, e quando integrada a equipe de saúde e a escola, aproxima o adolescente da temática, capacita os professores

nessa lacuna do conhecimento

e prepara os profissionais de

saúde para atuar próximo à realidade social. ver e qualidade de vida desses estudantes. Quando articulado com a Universidade e seus acadêmicos, permite extrapolar os "muros" entre ela e comunidade, fortalecendo seu papel enquanto formadora de atores sociais na realidade. Coube então, o seguinte questionamento: Quais estratégias o profissional de saúde, em particular o fisioterapeuta, pode utilizar no PSE para abordar a sexualidade, abuso e violência sexual?. Partindo disso, esse estudo objetiva relatar uma experiência sobre a importância da educação em saúde na escola para a conscientização da sexualidade na adolescência.

\section{MÉTODO}

Estudo qualitativo, descritivo e relato de experiência, de acadêmicos de fisioterapia, na Prática de Inserção da Fisioterapia na Comunidade I (PIFISIO I), da Universidade Federal de Sergipe (UFS), Campus Lagarto (SE) que possui acordo institucional junto ao município para realizar suas práticas e por isso, não houve aplicação de Termo de Consentimento Livre e Esclarecido (TCLE), mas respeitou-se os princípios éticos da RESOLUÇĀO $\mathrm{N}^{\circ}$ 466/2012 e RESOLUÇÃO No 510/2016 do Conselho Nacional de Saúde - CNS.

Utilizou-se o método do Arco de Charles Maguerez (observação da realidade, pontos-chave, teorização, hipóteses de solução e aplicação à realidade) que é utilizado na PIFISIO I e tem como objetivo gerar autonomia dos envolvidos, com conhecimento crítico e reflexivo, preparando-os para a realizar intervenções voltadas à necessidade de determinado território, seus sujeitos e atores sociais, possibilitando mudança dessa realidade. ${ }^{11}$ Para registro das etapas realizadas, o diário de campo no Sistema Integrado de Gestão de Atividades Acadêmicas (SIGAA) da UFS.

\section{RELATO DE EXPERIÊNCIA}

Todas as etapas desse relato ocorreram no período letivo 2018.1, entre outubro e novembro de 2018. Para a etapa inicial do arco, observação da realidade e elabo- 
ração dos pontos-chave, dialogou-se com uma escola municipal da região urbana do município de Lagarto (SE) e equipe de saúde de referência, respeitando-se os pilares preconizados pelo PSE e delimitou-se o público de maior impacto. Após o diálogo foi identificado o público do $6^{\circ}$ ano do ensino fundamental I, e a problemática: gravidez na adolescência e violência sexual. Com o propósito de aprofundar a temática e alicerçar o conhecimento dos acadêmicos (teorização e hipóteses de solução) realizou-se uma busca na base de dado da Biblioteca Virtual em Saúde (BVS), a partir dos descritores: "Educação em Saúde"; "Serviços de Saúde Escolar" e "Saúde sexual e reprodutiva", busca avançada, título, resumo e assunto. Para a seleção observamos se os artigos estavam de acordo com a temática nessa ordem: $1^{\text {a }}$ título, $2^{\circ}$ resumo, $3^{\circ}$ artigo e buscou-se durante a leitura identificar estudos que auxiliassem na associação da temática com a Fisioterapia, já que havia necessidade de associação com a prática de ensino da fisioterapia na comunidade.

Seguindo as etapas do arco (hipóteses de solução e aplicação à realidade) foi realizado o planejamento de duas intervenções, com intervalos para avaliação, discussão e diálogo com escola e equipe de saúde. Participaram das intervenções, 29 estudantes do ensino fundamental I, do $6^{\circ}$ ano, de 12 a 14 anos. Os assuntos abordados foram: anatomia do sistema reprodutor, fases do desenvolvimento corporal, sexualidade, fatores de risco para gravidez na adolescência e como identificar e prevenir a violência sexual. Assim, iniciou-se a construção de recursos e materiais didáticos, para serem utilizadas nas duas intervenções. A primeira com o intuito de proporcionar conhecimento e respeito acerca do corpo e suas alterações fisiológicas e a segunda para evitar casos abuso e/ou violência sexual, promovendo uma cultura de respeito e cuidado com o outro.

Na primeira intervenção, já com a sala organizada previamente pelos acadêmicos de fisioterapia, foi realizada uma dinâmica de acolhimento e apresentação sobre o motivo da presença de todos naquela ocasião. Foi proposta a construção de um cartaz coletivo, com uso de revistas, jornais, cartolinas, cola e tesoura, a partir de perguntas norteadoras, com o objetivo de ter acesso ao conhecimento prévio dos estudantes, acerca dos conceitos da sexualidade, abuso e violência sexual, se eram temas discutidos na escola, em casa ou com colegas, se havia diferenças entre homens e mulheres e se já haviam feito pesquisas na internet sobre as temáticas.

Dividiu-se a turma em dois grupos, com o auxílio dos facilitadores (acadêmicos e docente de fisioterapia), eles construíram seus cartazes e ao término, elegeram um representante de cada grupo para explicá-los, enquanto todos iam interagindo. Em seguida, os acadêmicos e a docente responsável sintetizaram os pontos colocados nos cartazes; usaram slides no powerpoint ${ }^{\circ} \mathrm{e}$ peças anatômicas, dos sistemas reprodutores masculino e feminino, do laboratório morfofuncional de anatomia da UFS para abordar a temática da sexualidade e a evolução biológica do corpo da criança, adolescente e adulto, e, apresentaram vídeos que abordavam a importância do cuidado e respeito com o corpo e seu autocuidado. Durante as apresentações e debate notou-se que muitos estudantes tinham dúvidas sobre o assunto e sugeriu-se que escrevessem no papel curiosidades geradas para serem trabalhadas no próximo encontro.

Para o segundo momento então, foi debatido a intervenção anterior e planejada uma peça teatral para demonstrar situações cotidianas, por meio de formas e tipos de abuso sexual, apresentando como pedir ajuda e informações sobre leis de proteção a favor da criança e adolescente: $\left(1^{a}\right)$ dois adolescentes que tiveram relação sexual sem preservativo gerando gravidez precoce, $\left(2^{\mathrm{a}}\right)$ assédio sexual dentro da escola cometido por colega de classe e $\left(3^{a}\right)$ professora explicando leis e formas de denunciar o abusador. Além disso, foram usados vídeos sobre prevenção, riscos e problemas da violência sexual e debate sobre a vivência. Ao final da intervenção buscou-se realizar uma avaliação acerca dos dois dias de intervenção e o que essas vivências tinham gerado em suas vidas.

\section{RESULTADOS E DISCUSSÃO}

No momento da construção do cartaz coletivo e apresentação dos conhecimentos prévios dos estudantes, foram observadas muitas curiosidades e diferentes olhares acerca da sexualidade, mas a maioria a associava ao termo "sexo", nesse contexto, restrito ao ato sexual propriamente dito, desconsiderando os diferentes aspectos da sexualidade. Muitos relataram diferenças no lidar com a sexualidade entre "homem e mulher" e preconceitos acerca de quem pode ou não fazer algo e da escolha sexual de colegas. Percebeu-se que a maioria consultava os colegas e tinham vergonha de usar a internet para pesquisar suas dúvidas sobre a temática. Além disso, relataram vergonha ou falta de "liberdade" sobre o assunto para falar com seus familiares. No âmbito da escola, poucos professores abordavam a temática, relataram apenas uma ação pontual de um professor de biologia, mas muitos ficaram com dúvidas. Durante o debate de abuso e violência eles identificaram situações em suas comunidades, o que demonstra a necessidade de se instituir políticas públicas voltadas para esse público e fortalecer esse debate

Durante o primeiro encontro notou-se que muitos estudantes tinham dúvidas sobre nomenclatura e funcionamento dos sistemas reprodutores, acerca dos cuidados e prevenções e do respeito com o outro e com seu corpo, e comportamentos de "vergonha" e "preconceitos" sobre a temática que se mostravam através de suas expressões corporais ou falas. Algo ainda importante, foi a presença de visão discriminatória quanto às escolhas sexuais de alguns estudantes que pode ser algo individual ou ocasionado pela instituição, pois muitas vezes os professores ou outros funcionários podem ter uma conduta discriminatória, apesar de não ter sido relatada nenhuma fala nesse sentido. Esses preconceitos podem ser ocasionados pela falta de informação, já que entre os temas apresentadas ao final desse encontro estavam: tipos de relacionamentos, escolhas sexuais e como identificar e agir em casos de abuso e violência sexual. 
Esses achados tornam importante ter como ponto de partida "o que eles sabem" e "o que eles podem fazer", desenvolvendo em cada um a capacidade de interpretar o cotidiano e atuar de modo a incorporar atitudes e/ou comportamentos adequados para a melhoria da qualidade de vida. ${ }^{3}$ Esse ponto de partida é fundamental, pois também evidencia o visualizado em outros estudos que trazem em seus achados alguns tabus, seja pelos estudantes, familiares e até da escola, sobre sexualidade, autocuidado, saúde sexual, prevenção de gravidez e IST/ AIDS e trazem estereótipos e crenças antigas sobre sexualidade e IST/AIDS. ${ }^{6,8,9}$.

Pode-se dizer que grande parte das pessoas entende a sexualidade como sinônimo de relação sexual. No entanto, outros fatores constituintes da sexualidade devem ser considerados, como aspectos psíquicos, sociais e culturais, distanciando-se do reducionismo biológico. ${ }^{4,12}$ Além disso, a crença de que falar sobre o tema estimula a curiosidade e pode antecipar práticas sexuais, deve ser ultrapassado, deve-se trabalhar sexualidade a partir de uma visão sócio-histórica, ressignificando normas e padrões vigentes de gênero e identidade, para fomentar o respeito, garantia dos direitos sexuais e a extinção de situações de preconceito e violência. Essas ações devem visar um espaço de diálogo, esclarecimento e problematização de questóes favorecendo a reflexão e ressignificação de informações, emoções e valores, principalmente no ambiente escolar. ${ }^{13}$

No que tange aos pais, esses assuntos são ainda pouco abordados pelas famílias, muitas vezes por dificuldade de falar sobre o assunto, seja por resistência devido à cultura ou à religião ou por falta de informação. Aos professores, traz um desafio ainda maior, pois esse "tabu" pode dificultar o diálogo e acrescido da carência de capacitação e incentivo pelas instituições para trabalhar o tema, pode levar ao uso de estratégias que aumentem o risco de condutas religiosas, repressivas, punitivas e discriminatórias e ainda marca uma negligência das escolas em relação à abordagem dessa temática. $\mathrm{E}$ percebe-se nos estudos, assim como neste que raramente ela é abordada. ${ }^{6,7,8,9,14}$
Tal fato demonstra a necessidade de abordar a temática na escola, pois os achados ratificam o fato de que a adolescência é envolvida pela ausência de informação adequada e negligência na busca deste conhecimento. Atrelado à falta de informação, as práticas sexuais ou relações sexuais tem início antes da fase adulta, de maturidade do saber, sem uso de preservativos $\mathrm{e}$, consequentemente, podem ocasionar infecções sexualmente transmissíveis, primeira relação sexual por pressão de colegas e o não reconhecimento de situações de abuso, violência e assédio sexual. ${ }^{15}$

Outro fato importante segundo Pesquisa Nacional de Saúde do Escolar (PeNSE), 2009, de estudantes do $9^{\circ}$ ano, é que $30,5 \%$ já tinham tido alguma relação sexual, sendo maior e mais cedo nas escolas públicas, e em dobro para o sexo masculino. Esse dado se torna importante, pois a idade da primeira relação é um dos indicadores mais usados para se analisar os riscos à saúde sexual e reprodutiva. ${ }^{4} \mathrm{E}$ por isso, os debates sobre sexualidade devem ser realizados com educadores, pais e profissionais da saúde, reduzindo a vulnerabilidade e comportamentos sexuais de risco. Alguns estudos nessa abordagem e que trazem a educação em saúde, mostraram melhora de vários aspectos relacionados à sexualidade, efetividade na diminuição da ocorrência e reincidência de gravidez na adolescência e prevenção de Doenças Sexualmente Transmissíveis (DST). ${ }^{9}$

De modo geral, nos estudos, as atividades desenvolvidas na temática da sexualidade, se caracterizam por intervenções temporárias, realizadas por profissionais que não pertencem à escola. Essas atividades atingem apenas uma parte da população escolar, concentrando $75 \%$ das ações no ensino fundamental $\left(5^{\circ} \text { ao } 9^{\circ} \text { ano }\right)^{7}$, como no caso no nosso estudo, em que foi escolhido pela instituição o $6^{\circ}$ ano, por considerarem de maior impacto da temática e foi um tema construído pelos acadêmicos de fisioterapia, de fora do PSE, apesar de estarem em debate com a escola e equipe de saúde.

Durante todas as etapas do arco foi perceptível a carência de preparo para abordar essa temática, seja pela escola quanto pelos profissionais de saúde que relataram carência de capacitação sobre o tema. Acerca dos profissionais de saúde, é fundamental essa inserção precoce na atenção básica, para dar-lhes segurança para liderar e administrar atividades de promoção de saúde e para que estabeleçam vínculo e responsabilidade pelo empoderamento de estudantes, professores e funcionários das escolas, devido ao impacto que podem trazer para a saúde da comunidade. ${ }^{16}$

Durante o segundo encontro, observou-se certo silêncio na sala e fixação no olhar dos alunos nos personagens apresentados, sendo perceptível emoção e surpresa. Quando questionados sobre as histórias, alguns identificaram as cenas em suas realidades como o fato de alguns meninos faltarem com respeito e limites de espaço, contra as meninas dentro do ambiente da escola. Aproveitou-se este e outros relatos para abordar a importância do respeito acerca da sexualidade.

Essa observação corrobora com os dados de notificação de violência contra adolescentes do Sistema de Informação de Agravos de Notificação, de 2011 a 2017, presente em $75,4 \%$ dos municípios, prevalecendo no sexo feminino (10 a 14 anos) e a negligência no sexo masculino (10 a 14 anos). ${ }^{17}$ Estudos internacionais apontam que a violência sexual é um fenômeno mundial e os impactos causados são diversos e afetam, mesmo a longo prazo, a vida dos sobreviventes, sendo negativos à saúde mental, física, sexual e reprodutiva deles. Assim, abordar o tema e o discutir, amplamente, em todas as esferas da sociedade é uma forma de mobilizar, sensibilizar e instrumentalizar o coletivo, desmistificando o assunto e chamando atenção para essa importante questão social. ${ }^{18}$

Ao final da intervenção, os escolares relataram "liberdade" para discutir a temática, essa relevância proporcionada pela metodologia da problematização possibilitou autonomia e protagonismo dos envolvidos, na construção, mediação e participação do conhecimento sobre os temas e foi uma oportunidade para desenvolver o senso crítico e reflexivo. Dessa forma, o conhecimento possibilita a formação de atores sociais conscientes de seus papéis de responsa- 
bilidade perante a sociedade e como podem reagir em relação à sexualidade. ${ }^{16}$

Pode-se dizer que a escola pode preparar o adolescente para o convívio no meio social, e por isso, é preciso analisar as percepções dos adolescentes sobre direito sexual e reprodutivo, tipos de violência, abuso e assédio sexual, para fundamentar uma construção com base na reflexão da autonomia e protagonismo do adolescente, usando ações educativas voltadas aos aspectos que facilitam a educação sobre este paradigma gerando agentes multiplicadores de conhecimento e cidadãos críticos com o exercício de direitos e deveres, capazes de controlar suas condições de saúde e qualidade de vida, com opção por atitudes mais saudáveis. ${ }^{19,3}$

\section{CONCLUSÃO}

A intervenção proporcionou uma conscientização acerca da sexualidade e dos cuidados contra o abuso e/ou violência sexual nos adolescentes da escola, permitindo uma quebra de paradigmas e "preconceitos", embutindo neles a importância do cuidado com seu corpo e o respeito com o outro e criando agentes multiplicadores capazes de gerar uma nova perspectiva de conhecimentos acerca da temática.

Aos acadêmicos de fisioterapia, a estratégia de ensino e aprendizagem utilizada possibilitou reflexão, estimulou a criatividade e a capacidade de atuação aumentando a compreensão da temática de forma dinâmica, ampliando o escopo de atuação da profissão e, ao interagir com a equipe de saúde e o Programa Saúde na Escola (PSE) fez com que as ações executadas tivessem papel significativo na abordagem de temas de caráter sociocultural e que geram mudanças na realidade social.

\section{REFERÊNCIAS}

1. Brasil CFF. A Saúde vai à escola: a promoção da saúde em práticas pedagógicas. Physis (Rio J.). 2015; 25(4):1207-27.

2. Brasil. Ministério da Saúde. Secretaria de Atenção à Saúde. Departamento de Atenção Básica. Cadernos de Atenção Básica, n. 26. Saúde sexual e saúde reprodutiva. 1. ed. Brasília: Ministério da Saúde; 2013.

3. Ministério da Saúde. Secretaria de Atenção à Saúde. Departamento de atenção básica. Cadernos de Atenção Básica n²4. Saúde na escola [internet]. Brasília: Ministério da Saúde. 2009. [acesso 2021 jan 29]. Disponivel em: http://bvsms.saude.gov.br/ bvs/ publicacoes/saude_escola_cab24.pdf.

4. Campos HM, Schall VT, Nogueira MJ. Saúde sexual e reprodutiva de adolescentes: interlocuções com a Pesquisa Nacional de Saúde do Escolar (PeNSE). Saúde Debate. 2013; 37(97):336-46.

5. Fontes LFC, Conceição OC, Machado S. Violência sexual na adolescência, perfil da vítima e impactos sobre a saúde mental. Cien Saude Colet. 2017; 22(9):2919-28.

6. Miranda CPV, Mororó RM, Rocha SMBS, Teodósio TBT, Silva MAM, Viana RS. Educação e saúde em sexualidade: Experiência com adolescentes do programa de erradicação do trabalho infantil. Rev. SANARE. 2017;16(02):103-8.

7. Furlanetto MF, Lauermann F, Costaiangel CB, Mariniv H. Educação sexual em escolas brasileiras: Revisão Sistemática da Literatura. Cad. pesqui. 2018;48(168):550-71.

8. Ataliba P, Mourão L. Avaliação de impacto do Programa Saúde nas Escolas. Psicol. esc. educ. 2018;22(1):27-36.

9. Barbosa LU, Machado RS, Pereira JCN, Lima AGT, Costa SS, Folmer V. Percepção de adolescentes sobre sexualidade e saúde reprodutiva: a escola como espaço para a educação sexual. Cult. cuid. 2019;23(55):25-34.

10. Viero VSF, Farias JM, Ferraz F, Simões PW, Martins JA, Ceretta LB. Educação em saúde com adolescentes: análise da aquisição de conhecimentos sobre temas de saúde. Rev. EEAN. 2015 jul;19(3):484-90.

11. Paulino DB, Rosa GFC, Alves GHF, Barros MCV, Oliveira BC,
Raimondi GA. Role-Play como Estratégia Pedagógica para Problematizar as Linhas de Cuidado Integral em Saúde aos Adolescentes e Jovens. Rev. bras. educ. med. 2019;43(1):662-71.

12. Nogueira MJ, Martins AM, Rodrigues AR. Escolas e unidades básicas de saúde: diálogos possíveis e necessários para a promoção da saúde sexual e reprodutiva dos adolescentes. Saúde Debate. 2012;36(92):117-24.

13. Ew RAS, Conz J, Farias ADGO, Sombrio PBM, Rocha KB. Diálogos sobre sexualidade na escola: uma intervenção possível.Psicol. pesq. 2017;11(2): 51-60.

14. Nothaft SCS, Zanatta EA, Brumm MLB, Galli KSB, Erdtmann BK, Buss E et al. Sexualidade do adolescente no discurso de educadores: possibilidades para práticas educativas. REME rev. min. enferm. 2014;18(2): 284-94.

15. Arruda EPT, Brito LGO, Prandini TR, Lerri MR, Reis RM, Barcelos TMR et al. Sexual Practices During Adolescence. Rev. Bras. Ginecol. Obstet. 2020 Nov;42(11):731-8.

16. Santos ACD, Gasparim CA, Monteiro GM, Brito MR, Silva VAM. Relato de Experiência: Construção e Desenvolvimento do Programa de Saúde na Escola (PSE) sob a Perspectiva da Sexualidade na Adolescência. Rev. bras. educ. med. 2019;43(4):193-9.

17. Pereira VOM, Pinto IV, Mascarenhas MDM, Shimizu HE, Ramalho WM, Fagg CW. Violências contra adolescentes: análise das notificações realizadas no setor saúde, Brasil, 20112017. Rev. bras. epidemiol. [Internet]. 2020 [acesso 2021 jun 13];23: E200004.SUPL.1. Disponivel em: 10.1590/1980549720200004.supl.1

18. Silva FC, Monge A, Landi CA, Zenardi, GA, Suzuki DC, Vitalle MSS. Os impactos da violência sexual vivida na infância e adolescência em universitários. Rev. Saúde Pública. 2020; 54(134):111.

19. Souza VP, Gusmão TLA, Frazão LRSB, Guedes TG, Monteiro EMLM. Protagonismo de adolescentes no planejamento de ações para a prevenção da violência sexual. Texto Contexto Enferm [Internet]. 2020 [acesso 2021 jan 21]; 29:e20180481. Disponível em: http://dx.doi.org/10.1590/1980-265X-TCE-2018-0481 